\title{
EDITORIAL
}

\section{Pelvic Organ Prolapse: From Basics to Newer Evolutions in Surgical Management of Uterine Prolapse}

\author{
Ruchika Garg ${ }^{1}$, Jaideep Malhotra² \\ Journal of South Asian Federation of Menopause Societies (2020): 10.5005/jp-journals-10032-1195
}

Prolapse is a common problem in women attending gynecological clinic. Normally when a woman strains, there is no descent of vaginal walls or of uterus. In prolapse, straining causes protrusion of vaginal walls at the vaginal orifice.

\section{Supports of Genital Tract}

\section{Delancey Levels of Support}

The levels of pelvic support are arbitrarily divided into three levels (Fig. 1). The understanding of this helps to localize the defect and thus, site-specific repair can be performed. ${ }^{1,2}$

- Proximal suspension by uterosacral-cardinal ligaments.

- Damage results in uterovaginal prolapse, posthysterectomy vaginal prolapse, and enterocele.

\section{Level II: Midvaginal Support}

- Lateral attachment to arcus tendenis fascia pelvic (ATFP) and ATFRV.

- Damage results in paravaginal and pararectal defects.

\section{Level III: Distal Vaginal Support}

- Distal fusion to urogenital diaphragm anteriorly and proximal perineum posteriorly.

- Anterior damage results in urinary incontinence and posterior damage results in perineal body deficits.

Cystocele and rectocele are due to central defects within pubocervical and rectovaginal septa, respectively.

At tissue level, it has been suggested that abnormal synthesis or degradation of collagen contributes to prolapse. The findings in women with prolapse are:

- Increase in total collagen content in vaginal wall.

- Immature collagen is more than mature collagen.

\section{Clinical Evaluation of Pelvic Organ DYSFUNCTION}

\section{Symptoms}

Common symptoms associated with pelvic organ prolapse are following:

- Sensation of something falling out.

- Pelvic discomfort, such as dragging sensation.

- Urinary symptoms.

- Urinary incontinence.

- Frequency.

- Urgency.

- Urinary retention.
${ }^{1}$ Department of Obstetrics and Gynecology, SN Medical College, Agra, Uttar Pradesh, India

${ }^{2}$ Rainbow IVF, Agra, Uttar Pradesh, India; South Asian Federation of Obstetric and Gynaecological Societies of India, Agra, Uttar Pradesh, India

Corresponding Author: Ruchika Garg, Department of Obstetrics and Gynecology, SN Medical College, Agra, Uttar Pradesh, India, Phone: +91 9720004485, e-mail: ruchikagargagra@gmail.com

How to cite this article: Garg R, Malhotra J. Pelvic Organ Prolapse: From Basics to Newer Evolutions in Surgical Management of Uterine Prolapse. J South Asian Feder Menopause Soc 2020;8(1):1-6.

Source of support: Nil

Conflict of interest: None

- Difficulty in emptying rectum.

- Sexual dysfunction.

- Decubitus ulcer.

- Leukorrhea due to congestion/inflammation.

\section{General Physical Examination}

It should include an evaluation of mental status, nutritional status, body mass index, anemia, and lymphadenopathy.

- Neurological system look for:

- Gait (pyramidal gait in upper motor neuron lesion, waddling gait in proximal muscle weakness, stamping gait in sensory dysfunction, foot drop in peripheral neuropathy).

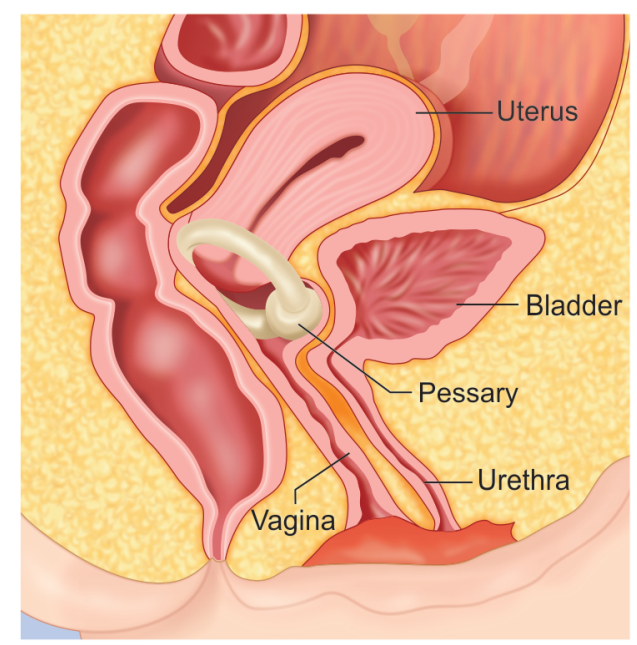

Fig. 1: Pelvic anatomy

(0) The Author(s). 2020 Open Access This article is distributed under the terms of the Creative Commons Attribution 4.0 International License (https:// creativecommons.org/licenses/by-nc/4.0/), which permits unrestricted use, distribution, and non-commercial reproduction in any medium, provided you give appropriate credit to the original author(s) and the source, provide a link to the Creative Commons license, and indicate if changes were made. The Creative Commons Public Domain Dedication waiver (http://creativecommons.org/publicdomain/zero/1.0/) applies to the data made available in this article, unless otherwise stated. 
- Spine (kyphotic, lordotic, or scoliotic abnormality, lipoma or tuft of hair in spina bifida, gibbus in skeletal tuberculosis).

- Joints-Range of motion.

- Muscle tone and power: hip flexion is controlled by motor segments of S2 and S3, plantar flexion is controlled by S1 and S2.

- Deep tendon reflexes.

- Superficial reflexes: Babinski reflex; fanning and dorsiflexion if corticospinal tracts are interrupted.

- Bulbocavernosus and anal wink reflex are elicited by gently stroking the perianal skin. Absence of these reflexes reflects the damage of pudendal nerve; however, in $10 \%$ of normal subjects, it is too week to visualize.

- Sensations: touch, cold, and prick, S2-S4 dermatomes of the perineum is evaluated.

- Abdomen: look for distension, free fluid, mass, scars, hernia.

\section{Local Genital Examination}

Position: after emptying the bladder, patient is asked to lie in dorsal lithotomy position. However, the semi-upright position would be a suitable to examine a case of prolapse. A mirror can be provided to the patient to confirm the maximum prolapse that is being observed on forceful straining. If not satisfactory, patient is to be examined in the standing position.

On inspection, comment on external genitalia including pubic hair, mons pubis, labia majora, clitoris, fourchette, scars, and tears. Patient is asked to maximally strain or cough. If seen, prolapsed mass can be of anterior or posterior vaginal walls or cervix, individually or in combination. Note should be made of any congestion, pigmentation, hyperkeratinization, and ulcers.

Decubitus ulcer should be recorded with location, dimensions, margins, edges, floor, sensations and relation to underlying structures, sloping edges with granulation tissue is suggestive of a healing ulcer. However, edges, such as punched out in syphilis, undermining in tuberculosis, and beaded in malignancy, are abnormal.

\section{Per-vaginal Examination}

A bimanual examination should be made to note the direction and size of uterus and adnexa. Levator tone should be appreciated with two fingers on the posterior vaginal at 5 or 7 o' clock position with thumb on the perineum and patient is asked to squeeze the pelvic muscle like holding the urine or avoiding passing of gas. It is graded according to modified Oxford scale.

- 0/5-Unable to contract.

- 1/5-Trace contraction, $<2$ seconds.

- 2/5-Weak contraction, $\geq 3$ seconds.

- 3/5-Moderate contraction, 4-6 seconds, posterior elevation of fingers, repeated three times.

- 4/5-Strong contraction, 7-9 seconds, posterior elevation of fingers, repeated four to five times.

- 5/5-Very strong contraction, $\geq 10$ seconds, posterior elevation of fingers, repeated four to five times.

\section{Per-rectal Examination}

Rectocele is confirmed by insinuating the finger on the anterior rectal wall toward vagina. Anal sphincter tone is graded on a scale of $0-4+$. With a thumb on perineum, the perineal body should be assessed for the bulk and integrity. Levator plate is a horizontal structure felt posterior to rectum which represents confluence of pelvic floor muscles.

Bidigital rectovaginal (PV and $\mathrm{RV}$ ) examination is performed to assess the thickness of rectovaginal septum. On straining if prolapsed mass is felt between the vaginal and rectal finger, it is indicative of enterocele.

\section{Evaluation of Bladder Function}

In patients with complaints of urinary incontinence, any leaking of urine with straining is documented. If present, then urethral hypermobility can be tested with a well-lubricated cotton swab stick inserted in the urethra up to $3 \mathrm{~cm}$ and asked to strain. If the angle of deflection is $>30^{\circ}$ from horizontal, it is indicative of loss of support at urethrovesical angle or urethral hypermobility. A protractor or a goniometer is used to measure the angle.

In severe prolapse, stress urinary incontinence can be masked due to kinking effect of the prolapse. Thus, prolapse should be reduced and patient is asked to strain with full bladder to note the leaking.

\section{Mapping of Tears}

- Rugosities on the vaginal walls are indicative of the underlying attached endopelvic fascia.

- Lateral vaginal sulci are the location of junction of pubocervical and rectovaginal to the respective arcuate attachments.

- The defects in the pubocervical septum can be proximal, distal lateral, or central. To map these tears, posterior vaginal wall is retracted with sim's speculum and the anterior prolapse mass is reduced with ring forceps posteriorly and cranially. If on straining, bulge is noted it is indicative central or combined central and paravaginal defects. If on straining, no prolapse is noted, the defect is mostly unilateral or bilateral paravaginal which can be detected by supporting each sulcus with closed ring forceps. If a bulge is seen just adjacent to the cervix in the anterior fornix, it is mostly due to apical detachment of pubocervical septum from pericervical ring.

- The defect in rectovaginal septum can be appreciated in the same way as described above. On per-rectal examination, the edge of torn septum can be felt.

- These tears are mapped on the pelvic organ prolapse map which aid in the site-specific repair. The most common defect is right paravaginal defect due to the predominance of left occipito anterior position of fetal head during labor.

\section{Investigations}

- Hemoglobin, hematocrit, total leukocyte count.

- Urine routine, microscopy, culture, and sensitivity.

- Blood sugar, urea, serum creatinine.

- CXR-PA view.

- Pap smear.

- Other investigations should be tailored to the clinical situation:

- If patient has abnormal uterine bleeding (AUB) then endometrial biopsy or aspiration must be performed.

- Urodynamic study may be required if urinary incontinence is not clinically defined.

- Decubitus ulcer biopsy in case of non-healing ulcers.

- Ultrasound KUB, intravenous pyleography (IVP) in case of hydronephrosis. 


\section{Anterior Vaginal Wall Defect}

- Anterior vaginal prolapse: It describes a defect of upper twothird of the anterior vaginal wall where urinary bladder is associated with prolapse. It is known as cystocele.

- Urethrocele: it is distal or lower third of anterior vaginal wall defect where urethra is associated with prolapse. It may be independent or usually along with cystocele the so-called cystourethrocele.

- Paravaginal/midline/transverse prolapse: it indicates the location of anterior vaginal wall defect.

\section{Apical Prolapse}

- Uterine prolapse.

- Vaginal vault prolapse (posthysterectomy or secondary vault prolapse).

- Enterocele: it describes an apical vaginal wall defect in which bowel or omentum or both are contained within the prolapsed segment. Traction enterocele is secondary to uterovaginal prolapse. Pulsion enterocele is secondary to chronically raised intra-abdominal pressure.

\section{Posterior Vaginal Wall}

- Relaxed perineum: torn perineal body produces gaping introitus with bulge of the lower part of the posterior vaginal wall.

- Rectocele: there is laxity of the middle one-third of the posterior vaginal wall.

\section{Differential Diagnosis}

Prolapse of vagina is generally a straightforward diagnosis. However, less common disease entities may present as bulge in the vagina:

- Tumors of urethra and bladder are much more indurated and fixed then anterior vaginal prolapse.

- Urethral diverticulum is usually more focal and painful. Compression may express some purulent material from the urethral meatus. Urethroscopy helps in diagnosis.

- Anterolateral defect can represent embryological remnants as Gartner's duct cyst.

- Skene's and Bartholin gland can enlarge to form cyst or abscess.

- Congenital elongation of cervix is differentiated from the POP as vaginal portion of the cervix is elongated and there is no accompanying vaginal prolapse. The fornices are deep, not shallow.

- Cervical fibroid polyp can be identified as cervix is high up in its normal anatomical position.

- Chronic inversion: It is recognized as cervix is further up, uterus cannot be defined. Uterine sound will confirm the diagnosis. On laparoscopy, there will be fundal depression and absence of uterine fundus in the pelvis.

\section{Conservative Measures}

\section{Primary Treatment}

Pessaries are supportive vaginal devices, usually made from inert material, such as silicone. They are designed to be placed into the vagina, to retain and hold pelvic structure in their normal positions. Pessaries are used when surgery is not elected or contraindicated.
Table 1: Common surgical procedure for treatment of pelvic organ prolapse

\begin{tabular}{lll}
\hline Defect location & Clinical condition & Procedure \\
\hline Anterior & Cystocele & $\begin{array}{l}\text { Anterior colporrhaphy } \\
\text { paravaginal repair } \\
\text { Superior (apical) }\end{array}$ \\
& Uterine prolapse & $\begin{array}{l}\text { Hysterectomy (Fig. 2) } \\
\text { Colpocleisis }\end{array}$ \\
& Eaginal enterocele repair \\
& McCall culdoplasty \\
& & Abdominal enterocele \\
& & repair \\
\hline
\end{tabular}

They can also be used as temporary treatment in a patient awaiting a surgical procedure. Pessaries are effective in relieving symptoms of POP (Table 1).

\section{Paravaginal Repair}

Confirmed preoperatively by noting loss of the lateral sulci and lack of rugation over the epithelium along the base of the bladder and elongation of the anterior vaginal wall.

Clinically, vaginal examination using a speculum reveals a preponderance of the prolapse lateralized to one side as speculum is withdrawn. Besides this, a ring forceps can be used by gently exerting anterior traction along the vaginal sulci. If the defect is reduced then the defect is peravaginal and can be approached with a paravaginal repair technique.

In these cases, repair should be performed by fixing the endopelvic fascia to the arcus tendineus fascia (white line) of the pelvis. This may be performed retropubically through the space of Retzius or vaginally.

\section{Traditional Repair}

After separating the vaginal epithelium off the underlying rectovaginal fascia as in traditional method, the surgeon inserts a finger of nondominant hand into the rectum to inspect the rectovaginal fascia for defect. These defects are then repaired with interrupted suture to plicate over the rectal wall. In this levator ani is not plicated as this may result in band-like stricture over the posterior wall, causing dyspareunia.

Perineorrhaphy is generally combined with posterior vaginal repair. This procedure restores the perineal body and reduces the vaginal outlet to more normal caliber. Reapproximation of the superficial transverse perineal muscle and bulbocavernosus muscle rebuilds the perineum and lengthens the distance between vaginal opening and anal verge.

\section{Apical Vaginal Repair}

The vaginal apex can be resuspended with a number of procedures:

- Sacrospinous ligament fixation.

- Uterosacral ligament vaginal vault suspension.

- Abdominal sacropexy.

\section{Technique}

Sacrospinous ligament is identified $2-3 \mathrm{~cm}$ medial to ischial spine and two or more unabsorbable or delayed absorbable ligature is passed through the ligaments to the submucosal apex of the vagina. Tying the suture brings the vaginal apex to sacrospinous ligament and posterior colporrhaphy is then performed. 

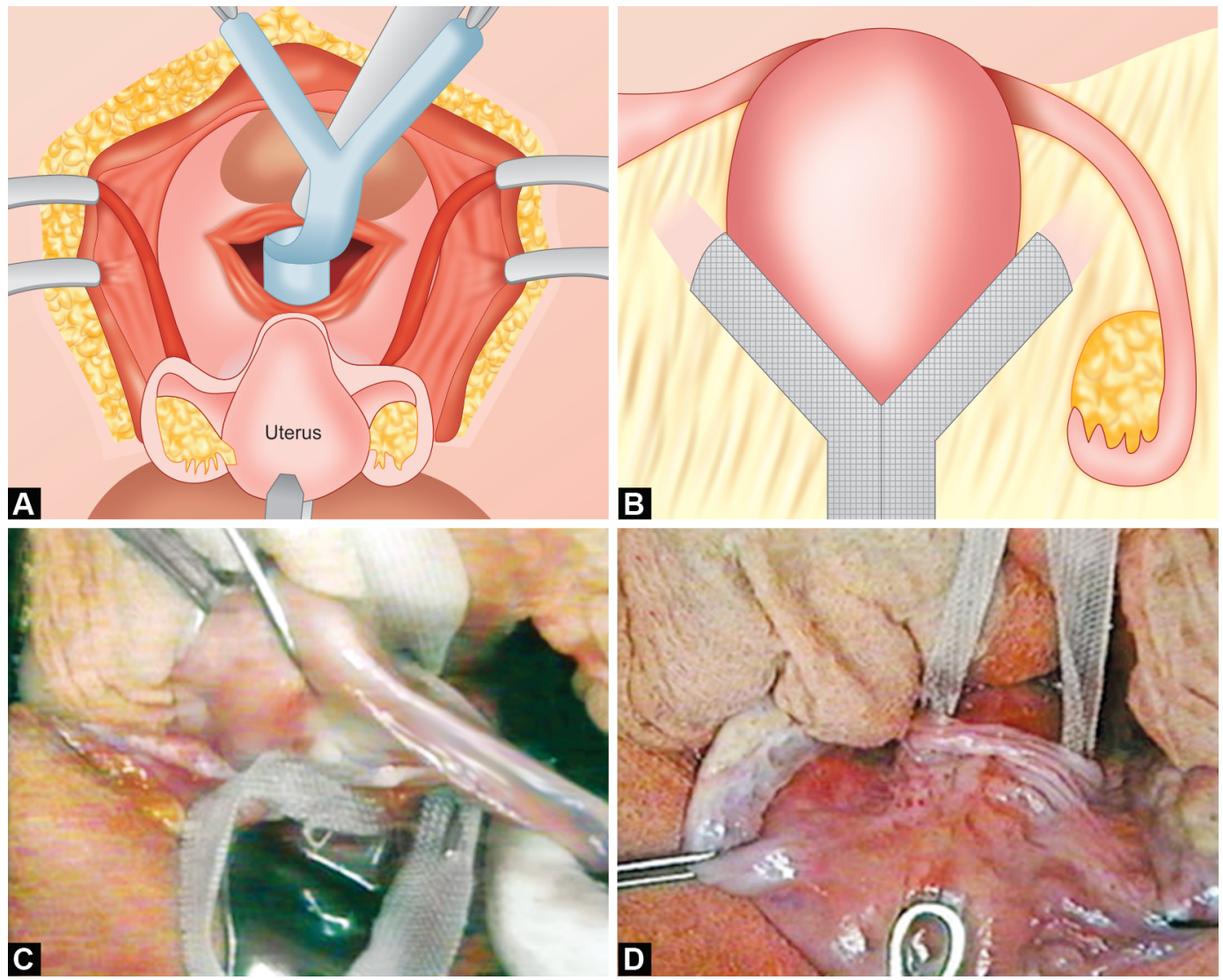

Figs 2A to D: Sacrocolpopexy for vaginal vault prolapse

\section{Bilateral Uterosacral Ligament Suspension}

This technique can be performed at the time of vaginal hysterectomy or to correct post-hysterectomy apical cuff prolapse. After entrance into the peritoneum is complete, traction on the ipsilateral posterior vaginal wall with rectal digital examination will facilitate transperitoneal identification of the uterosacral ligaments. Now two sutures with non-absorbable material in lateral to medial fashion is placed-one at the level of ischial spine and another a little high up on both sides. These sutures are then brought to the ipsilateral vaginal apices. Fixation of the cuff at this level reproduces cuff placement to the normal position of the cervicovaginal junction. Anterior vaginal repair should be performed prior to tying down the vaginal cuff.

\section{Abdominal Sacrocolpopexy}

It is the procedure of choice for those who are already having an abdominal approach for hysterectomy or for other indication

By laparotomy or by laparoscope cul-de-sac and peritoneum overlying the sacrum is visualized. A window in the peritoneum over sacral promontory is created and permanent sutures are placed through the anterior longitudinal ligament, approximately at level of S1. The vaginal cuff is then exposed by dissecting off the overlying peritoneum. Fixation of Y-shaped graft over anterior and posterior vagina is performed. This Y-shaped graft is then brought posteriorly along the hollow of sacrum and affixed to the anterior longitudinal ligament suture, overriding the sacral promontory.

Many different graft types have been described. Biological grafts have high-failure rate when placed at apex. As graft technologies evolve, identification of the optimal graft material then maximizes durability and compatibility may materialize. Besides graft complications, operative hemorrhage is significant risk. Specifically during placement of sacral suture laceration of sacral vein may be problematic.

\section{Obliterating Vaginal Operation \\ Colpocleisis and Le Fort's Operation}

These procedures involve removing excessive vaginal epithelium, suturing anterior and posterior wall together, obliterating the vaginal vault, and effectively closing the vagina.

\section{Forthergill's Repair (Manchester Operation)}

In this anterior and posterior colpoperineorrhaphy is combined with amputation of cervix, suturing of cut end of Mackenrodt's ligament in front of the cervix and covering raw area of amputated cervix with vaginal mucosa (Strumdorf's suture). This operation is suitable for women in reproductive age group. However, fertility is somewhat reduced and there can be incompetent cervical os and resultant risk of abortion and preterm delivery.

\section{Vaginal Hysterectomy}

Vaginal hysterectomy with anterior and posterior colpoperineorrhaphy is recommended in clinical practice over age of 40 years, who are not keen on retaining their childbearing and menstrual function. If there is associated abnormal uterine bleeding, fibroid, adenomyosis this age can be relaxed up to 35 years (Fig. 2). However, in European countries, hysterectomy is 
rarely performed during pelvic floor reconstruction while in United States and India hysterectomy is often performed concurrently with prolapse surgery.

If apical or uterine prolapse is present, hysterectomy will more readily allow the vaginal apex to be suspended with the abovedescribed apical suspension procedures. Alternatively if apical or cervical prolapse is not present, hysterectomy need not to be incorporated into prolapse repair.

\section{Procedure of Vaginal Hysterectomy}

A cervical incision is performed and the uterovesical fold and pouch of Douglas opened. The uterosacral and cardinal ligament are divided and ligated first, followed by uterine pedicles and finally the tubo-ovarian and round ligament. The sequence can be reversed also, according to ease of surgeon. In case of procidentia, care should be taken to avoid kinking of ureters which are often dragged into lower positions than normal. After closure of the pelvic peritoneum, the upper pedicles are tied in the midline to give support for the vaginal vault. In the same way, uterosacral ligaments are tied posteriorly to obliterate potential enterocele space. The vaginal epithelium is then closed with interrupted sutures.

\section{Newer Advances in Surgical Treatment of Pelvic Organ Prolapse}

The new surgery aims to create new connective tissue to replace broken ligaments and septa instead of trying to tighten or suture an altered suspensory apparatus. Synthetic meshes or biological grafts are used for this purpose. Anatomy is restored rather than distorted, and postoperative pain is tremendously reduced.

\section{Transvaginal Mesh}

Almost $30 \%$ of patients will require a reoperation indicating weakness in native tissue repairs.

Standard cystocele repair carries a high-failure rate. Recurrence risk with prolapse repair appears to be significant in the anterior compartment compared to the apical and posterior.

\section{Mesh Use in Prolapse Surgery}

The First-generation Needle-driven Kits

Anterior compartment: Perigee (American Medical System) used helical needles through the obturator foramen to place a new hammock-type polypropylene mesh. The principle was that it would effectively address multiple defects in the fascial hammock.

Anterior PROLIFT (Johnson and Johnson, New Jersey) worked on a similar principle.

\section{Second-generation Mesh Kits}

Elevate system (American Medical System) and pinnacle (Boston Scientific).

These use a single vaginal incision for both dissection and introducing the mesh device. These mesh kits use trocar-less delivery systems and lighter meshes. These devices obviate the need to use blind needle pass and thereby reduce complication rates related to insertion.

\section{Complications}

The most common complications are mesh exposure (erosion) and pain (including dyspareunia). Deaths have been reported in association with bowel perforation and hemorrhage.
Exposure (erosion) may occur with any reconstructive material (e.g., synthetic or biograft) and following any pelvic reconstructive procedure. Patients with mesh exposure may be asymptomatic.

Contraction: contraction of mesh appears to cause painful vaginal bands resulting in dyspareunia.

Pelvic pain or dyspareunia: pelvic pain following transvaginal mesh (TVM) placement can occur even in the absence of mesh contraction pain has been reported in up to $30 \%$ of patients.

Infection: urinary tract and vaginal wound infections are the most common types of infection following TVM placement. Urinary tract infection rates $8-26 \%$ : vaginal wound infection rates $1-18 \%$

Abscess formation (pelvic, retropubic, or other sites) can occur following transvaginal placement of reconstructive material.

Voiding dysfunction: $7-12 \%$.

Overactive bladder symptoms and urgency urinary incontinence were $18 \%$.

Other complications: granuloma formation 3-39\% visceral injury (e.g., bladder, rectal, and vaginal perforation) 1-14\%. Bleeding complications $0-3 \%$, fistula formation $1 \%$ in POP repair.

In January 2012, the FDA issued orders requiring mesh device manufactures to perform post-market surveillance studies.

\section{Recommendations for Using Mesh}

- Recognize that in most cases POP can be treated successfully without TVM thus avoiding the risk of mesh-related complications.

- Choose TVM surgery only after weighing the risks and benefits of surgery with mesh vs all surgical and non-surgical alternatives.

- Consider these factors before placing surgical TVM:

- Surgical TVM is a permanent implant that may make future surgical repair more challenging.

- A TVM procedure may put the patient at risk for requiring additional surgery or for the development of new complications.

- Removal of TVM due to mesh complications may involve multiple surgeries and significantly impair the patient's quality of life. Complete removal of mesh may not be possible and may not result in complete resolution of complications including pain.

- Transabdominal mesh POP repair may result in lower rates of mesh complications compared with TVM POP surgery.

- Inform the patient about the benefits and risk of non-surgical options, suture-only surgery, surgical mesh placed abdominally, and the likely success of these alternatives compared with TVM placement.

- Ensure that the patient understands the postoperative risk and complication of TVM surgery as well as crucial document a discussion of: know risks exposure; erosion; early or delayed; fistula formation; chronic pain; dyspareunia; infection; recurrent prolapse; new or persistent incontinence; possible future surgery; long-term durability data vs native tissue repair.

The key points for preventing complications from TVM are:

- Optimization of modifiable risk factors (smoking-cessation therapy is encouraged, vaginal atrophy and ulceration; preoperatively, patients are treated with topical estrogen until the symptoms resolve.

- Selection of the mesh type based upon biomechanical properties of the material. Macroporous polypropylene mesh is the most SUI and for abdominal mesh repairs. Materials with microporous structure are associated with increased complications rates and should be avoided. 
- Adequate surgical training.

- Full-thickness dissection with development of a deeper surgical plane.

- Tension-free mesh suspension: the mesh needs to be inserted in a tension-free fashion. Excessive tension in the arms has been associated with tight vaginal bands, pain and exposure due to mesh contraction.

- Prevention of mesh rolling or bunching: the mesh body needs to be trimmed and secured to the underlying tissue to prevent rolling or bunching, as this phenomenon has been observed in patients who experience mesh exposure and pain.

Best kind of vaginal mesh

- Consider a trocar-less system or surgeon-styled graft cut to fit.

- Place as little material as possible to address the patient's support defect.

- Use the system with which you are most comfortable.

\section{SUMMARY}

Mesh was introduced to reduce the risk of recurrent prolapse. It has helped anatomically but symptomatically has not helped. As far as reoperation rate is concerned, it has not helped.

\section{Laparoscopy/Robotics}

Laparoscopic surgery has some potential advantage:

- Improved anatomic visualization of the peritoneal cavity, presacral apace, and space of Retzius, which can be attributed to laparoscopic magnification, insufflation effects, and improved hemostasis.

- Shortened postoperative hospitalization resulting in potential cost reduction, decreased postoperative pain, more rapid recovery and return to work, and better cosmetic appearance of smaller incisions.

Laparoscopic surgery has some potential disadvantage:

- Prolonged learning curve.

- Increased operating time.

Laparoscopic uterine suspension procedures (e.g., uterosacral ligament uterine suspension or sacrocervicopexy) may be considered when there is stage II to III prolapse (POP-Q classification), as an alternative to hysterectomy.

- Consider uterosacral ligament uterine suspension if intraoperative evaluation demonstrates adequate integrity of the uterosacral ligaments, and reserve the more complicated sacrocervicopexy foe patients with attenuated uterosacral ligaments, or for those with more advanced prolapse who desire uterine preservation. Identify the proximal portion of the uterosacral ligament at the level of the ischial spine and use permanent sutures to bind it to the distal aspect of the ligament near its insertion into the lower uterine segment and cervix. This reapproximation of the avulsed or attenuated ligament restores level I support of the proximal vagina in a horizontal position over the levator plate. The use of a uterine manipulator may assist with identification of the uterosacral ligaments by deflecting the uterus sharply to the contralateral side.

The pelvic ureter must be identified before placing sutures in the uterosacral ligament. If needed, a releasing incision may be made in the peritoneum just lateral to the ligament (and medial to the ureter) to prevent kinking of the ureter.

- Sacrocervicopexy involves suturing the posterior vagina and cervix to the sacrum using an intervening graft, which may be either synthetic (e.g., polypropylene and polyester) or biological (e.g., fascia and dermis).

- "Y-shaped" mesh in which one mesh segment is attacked to the posterior rectovaginal fascia while another mesh segment extends over the cervix and may be used to support the anterior pubocervical fascia.

- Vaginal vault prolapse can be repaired laparoscopically by synthetic mesh (polypropylene or polyester).

\section{Sacrocolpopexy}

Duplicate the steps performed in the open procedure, as closely as possible.

To perform laparoscopic sacrocolpopexy, after the bladder and rectum are dissected off the anterior and posterior vaginal walls, respectively, a Y-shaped synthetic graft is sutured to the anterior and posterior and pelvic fascia with a series of permanent sutures. The graft is fixed to the anterior longitudinal ligament of the sacral promontory, after dissection is performed in the presacral space. Burying the grant under the peritoneum.

Most rectoceles can be treated via a vaginal approach by site-specific repairs. Laparoscopic repair has been described as an alternative approach.

\section{Advantages of Robotic Surgery}

- Superior visualization.

- Mechanical improvements.

- Stabilization of instruments within the surgical field robotassisted surgery minimizes surgeon tremor.

- Improved ergonomics for the operating surgeon.

\section{Limitations of Robotic Surgery}

- Additional surgical training.

- Increased costs and operating room time.

- Bulkiness of the device: once the robotic system is docked, the patient bed position cannot be changed without first removing instruments and undocking the robotic arms.

- Instrumentation limitations (e.g., lack of a robotic suction and irrigation device size and cost).

- Lack of tactile feedback: the surgeon has to pay close attention to visual cues when placing tension on tissues or suture.

- The tip of the robotic endoscopic camera becomes very hot and must be cleaned outside of the peritoneal cavity.

- Risk of mechanical failure.

- Limited number of energy sources (less than with conventional laparoscopy).

\section{References}

1. Olsen AL, Smith VJ, Bergstrom JO, et al. Epidemiology of surgically managed pelvic organ prolapse and urinary incontinence. Obstet Gynecol 1997;89(4):501-506. DOI: 10.1016/S0029-7844(97)00058-6.

2. Weber AM, Walters MD, Piedmonte MR, et al. Anterior colporrhaphy; a randomized trial of three surgical techniques. Am J Obstet Gynecol 2001;185(6):1299-1304. DOI: 10.1067/mob.2001.119081. 